\title{
Dengue Hemorrhagic Fever with Discolored Leg Syndrome in a Neonate: Authors' Reply
}

\author{
Manoharan Vijoo • Ramalingam Raja • Chandrasekaran Venkatesh • \\ Uthirakumaran Ramkumar • Palanisamy Soundararajan
}

Received: 21 October 2014 / Accepted: 5 November 2014 / Published online: 23 November 2014

(C) Dr. K C Chaudhuri Foundation 2014

To the Editor: We thank Professor Ghosh for comments on our paper and emphasizing on the role of FDP, D-Dimer and serum fibrinogen in diagnosing DIC [1]. In our case, we ruled out bacterial sepsis with DIC as a cause by a negative CRP and blood culture in a stable non sick looking neonate [1]. The inadvertent error which led to the misunderstanding is regretted. Dengue infection can be complicated by DIC in patients with severe illness who present with shock and organ failure [2]. However DIC as the cause of acral discoloration is unlikely because of the following reasons:

The neonate was hemodynamically stable with good peripheral circulation and did not require intensive care support. For DIC to cause acral discoloration, there should be evidence of thrombus formation in vessels of the distal areas of the leg and foot which was ruled out by presence of good peripheral pulses and Doppler pulsatility. If the acral discoloration was truly associated with DIC (which then would be due to thrombosis), the discoloration would not have disappeared without starting specific therapy like heparin, which was not done in the index case. In contrast, the leg discoloration was the first to improve compared to other areas of echymosis and purpura, suggesting that it may not be a truly thrombo-hemorrhagic phenomena. Finally, thrombocytopenia, prolonged PT and aPTT, elevated liver enzymes can all be explained by dengue virus infection itself. The leg discoloration was probably due to a vasogenic cause and may be a part of dengue vasculopathy. More studies are needed to ascertain the cause.

Conflict of Interest None.

Source of Funding None.

\section{References}

1. Vijoo M, Raja R, Venkatesh C, Ramkumar U, Soundararajan P. Dengue hemorrhagic fever with discolored leg syndrome in a neonate. Indian J Pediatr. 2014;81:833-4.

2. Kamath SR, Ranjith S. Clinical features, complications and atypical manifestations of children with severe forms of dengue hemorrhagic fever in South India. Indian J Pediatr. 2006;73:889-95. 